\title{
Approches d'apprentissage et réussite en première année commune des études de santé (PACES) en France
}

\section{Learning Approaches and Selection in the Common First Year of Health Studies in France}

Marie-Paule GUSTIN ${ }^{1}$, Christine VINCIGUERRA ${ }^{1,2}$, Siara ISAAC ${ }^{3}$, Carole BURILLON ${ }^{2,4}$ et Jérôme ETIENNE ${ }^{2,5}$

1 Faculté de Pharmacie (Institut des sciences pharmaceutiques et biologiques) de Lyon, Université Claude-Bernard Lyon 1, Lyon, France

2 Hospices Civils de Lyon, Lyon, France

3 Service Innovation, Conception et Accompagnement pour la Pédagogie (ICAP), Université Claude-Bernard Lyon 1, Lyon, France

4 Faculté de médecine Lyon SUD, Université Claude-Bernard Lyon 1, Lyon, France

5 Faculté de médecine Lyon EST, Université Claude-Bernard Lyon 1, Lyon, France

Manuscrit reçu le 4 mars 2016 ; commentaires éditoriaux formulés aux auteurs le 4 avril 2016 ; accepté pour publication le 24 avril 2016

\section{Mots-clés}

Première année commune des études de santé ; Approche d'apprentissage ; Réussite ; Concours ; Sélection
Résumé - Contexte : Mise en place en France en septembre 2010, la Première Année Commune des Etudes de Santé (PACES) est caractérisée par un enseignement de masse, un concours très sélectif et une grande quantité d'information à mémoriser. Dans ce contexte très particulier, nous avons, pour la première fois, déterminé les approches d'apprentissage spécifiquement adoptées par les étudiants et étudié leur impact sur la réussite au concours. Matériel et méthodes : Une version française du questionnaire R-SPQ-2F de Biggs a été administrée aux étudiants de PACES 2011-2012 avant les partiels en décembre. Les approches d'apprentissage spécifiques ont été déterminées après analyse factorielle exploratoire du R-SPQ-2F et sélection des items pertinents dans ce contexte. L'association entre les approches et la bonne réussite aux partiels a été étudiée par un modèle de régression binomiale. Résultats : Au total, 1977 étudiants sont inclus dans l'analyse. Les étudiants qui ont le plus de chance de réussir sont i) ceux qui s'intéressent à leurs études (risque relatif (RR) de réussite de 1,5) et ii) ceux qui se limitent au contenu du cours (approche de surface, $R R=1,4$ ). Les étudiants qui apprennent par cœur (approche de surface) ont 1,6 plus de risque d'échouer que les autres. Conclusion : Ce concours de PACES très sélectif et très stressant a pour effet d'encourager une certaine approche de surface très forte en vue de la réussite au concours au détriment d'une approche plus en profondeur indispensable à une formation solide. 


\section{Keywords}

Common first year of health studies; Learning approach; Academic achievement; Selection for medical studies

\begin{abstract}
Context: The common first year of health studies (PACES) was implemented in September 2010 in France. First-year classes are quite large. There is also a significant amount of information to memorize and a selective highly competitive final exam. In this special context, we implemented learning approaches adopted by students and studied their impact on academic achievement. Material and methods: A French translation of John Biggs' R-SPQ-2F questionnaire was administered to PACES students before the mid-term exams in December 2011. Specific learning approaches were determined following an exploratory factor analysis and a selection of relevant items in the context. A binomial regression model was used to assess the association between approaches and high grades at mid-term exams. Results: Overall 1.977 students were included in the analysis. Students with greater chances of success were the ones who i) were interested in their studies (relative risk $(\mathrm{RR})$ of success $=1.5$ ) and ii) focused exclusively on course material (surface approach, $\mathrm{RR}=1.4$ ). Students who learned by heart (surface approach) were 1.6 times more at risk to fail than the others. Conclusion: This very selective and stressful exam benefits students who adopt strong surface approaches in order to succeed rather than develop in-depth approaches involving thorough training.
\end{abstract}

\section{Introduction}

\section{Contexte}

La réforme de la première année commune des études de santé (PACES) mise en place en France en septembre 2010 subit aujourd'hui de nombreuses controverses, liées à la façon dont les étudiants abordent leurs études, notamment au regard des activités d'apprentissage qu'ils développent dans ce cadre.

Evaluer la façon dont les étudiants abordent leurs tâches d'apprentissage est complexe et a donné lieu à de nombreux travaux. Issus des analyses qualitatives de Marton and Säljö ${ }^{[1,2]}$ ces travaux ont permis de faire émerger le concept d'approche d'apprentissage (approach to learning ou learning approach). Les modèles d'approche d'apprentissage décrivent le comportement adopté par l'étudiant face à une tâche d'apprentissage spécifique. Ce comportement dépend à la fois de l'étudiant (acquis antérieurs, compétences, prédispositions) et du contexte pédagogique (nature de la tâche, méthodes d'enseignement et procédures d'évaluation, nature de l'établissement). Deux modèles proviennent de travaux ayant suscité beaucoup d'intérêt : 1) les travaux $\mathrm{d}^{\prime}$ Entwisle ${ }^{[3]}$ (Approches and Study Skills Inventory for Students -ASSIST, 1998) et 2) les travaux de
Biggs $^{[4]}$ (Study Process Questionnaire -SPQ, 1978). Après plusieurs modifications importantes depuis sa publication, le SPQ est composé, en 1982, de 42 items et trois dimensions : approche profonde (deep approach), approche de surface (surface approach) et approche pour la réussite (achieving). Biggs l'a de nouveau simplifié en ne conservant que l'approche profonde et l'approche de surface (Revised Two Factors Study Process Questionnaire, R-SPQ-2F, 2001) ${ }^{[5]}$.

Ce questionnaire R-SPQ-2F est très largement utilisé dans le domaine universitaire. Il est décrit comme sensible au contexte pédagogique et cultu$\mathrm{rel}^{[6-9]}$. Il vise à distinguer motivation (pourquoi je travaille) et stratégie (comment je travaille) pour des approches d'apprentissage en profondeur et en surface. Ainsi, dans le cas de l'approche profonde mesurée par le R-SPQ-2F, la motivation est interne et se traduira par l'intérêt, le plaisir d'apprendre ; la stratégie consistera en un travail personnel d'approfondissement, de compréhension et de synthèse. Dans le cas de l'approche de surface, la motivation est externe et vise la réussite en étant efficace; la stratégie consiste à apprendre en fonction des modalités de contrôle des connaissances. Deux façons de faire et d'être : d'un côté le plaisir d'apprendre pour comprendre et dominer une question et de l'autre le sentiment d'être rapide et efficace en terme de réussite aux examens. 
Il est important de souligner qu'il est inapproprié de cataloguer un étudiant comme profond ou superficiel, comme s'il s'agissait d'un trait de sa personnalité, car la tâche et le contexte de la situation d'apprentissage ont une forte influence sur l'approche de l'étudiant.

On peut supposer que des étudiants en santé travaillant en profondeur seront plus autonomes dans leur apprentissage et mieux préparés à la formation continue ultérieure $^{[10,11]}$. De plus, des travaux antérieurs montrent que la façon de travailler adoptée par les étudiants dépend beaucoup du contexte de formation en particulier de la forme des cours et du type d'évaluation ${ }^{[12,13]}$. Il est ainsi nécessaire de pouvoir évaluer la propension des étudiants à travailler en profondeur ou en surface, ces deux pratiques n'étant pas forcément exclusives ${ }^{[5,14]}$.

Le modèle d'interprétation proposé par Biggs peut être nuancé par des modèles plus fins. Ainsi, certains auteurs ont pu préciser certains aspects des approches précédemment citées comme «s'intéresser aux études », « apprendre par cœur », « fournir du travail personnel », « travailler avec le minimum d'effort ${ }^{[7,9]}$. Ces approches d'apprentissages fines sont potentiellement intéressantes dans des contextes très spécifiques comme celui de la PACES. Il nous a donc semblé important d'analyser l'association entre les approches d'apprentissage fines adoptées par les étudiants et leur réussite en PACES afin de déterminer si certaines approches nécessaires à la réussite ne risqueraient pas d'être néfastes pour la suite de la formation.

\section{Objectif}

L'objectif de ce travail est double :

1) analyser la structure factorielle du R-SPQ-2F de Biggs au niveau des items dans le contexte de la PACES afin de déterminer quelles approches fines peuvent être mises en évidence avec la version française de ce questionnaire,

2) étudier les associations de ces approches avec la réussite aux examens partiels du concours de PACES en fin du premier semestre.

\section{Méthodes}

\section{Participants}

Les étudiants de PACES inscrits en septembre 2011 ont été recrutés pour cette étude. L'administration du questionnaire (entre le 8 et le 30 novembre 2011), effectuée en ligne, a été proposée de façon systématique lors de l'entrée de l'étudiant dans le module de PACES de la plateforme pédagogique de l'Université Lyon 1. De plus, l'étudiant devait répondre à l'item en cours afin de pouvoir passer à l'item suivant. Les examens partiels du concours de PACES ont lieu mi-décembre.

\section{Instrument : le R-SPQ-2F de Biggs}

Ce questionnaire, donné en annexes 1 à 3 , comprend 20 items avec 10 items par dimension (approche profonde et approche de surface) composée chacune de cinq items pour chacune des sousdimensions (motivation en profondeur -MP-, stratégie en profondeur-SP-, motivation de surface -MSet stratégie de surface-SS-). La structure complète à quatre sous-dimensions et 20 items du R-SPQ-2F proposée par Biggs est donnée figure 1. Les étudiants répondent à chaque item en sélectionnant une réponse catégorielle fréquentielle sur une échelle allant de 1 (pour la réponse $\mathrm{A}$ ) à 5 (pour la réponse $\mathrm{E})(\mathrm{A}$ : cet item n'est jamais ou seulement rarement vrai pour moi ; B : cet item est vrai de temps en temps pour moi ; $\mathrm{C}$ : cet item est vrai environ une fois sur deux pour moi ; D : cet item est souvent vrai pour moi ; E : cet item est toujours ou presque toujours vrai pour moi).

En collaboration avec l'Unité de développement et de recherche en éducation médicale de l'Université de Genève, les consignes et le questionnaire original ont été traduits en français puis retraduits en anglais par un deuxième traducteur qui ne connaissait pas la version originale afin de détecter d'éventuelles incohérences entre les deux versions. La version anglaise du questionnaire est également donnée en annexe 3 et les consignes (en français et en anglais) en annexe 4 . 


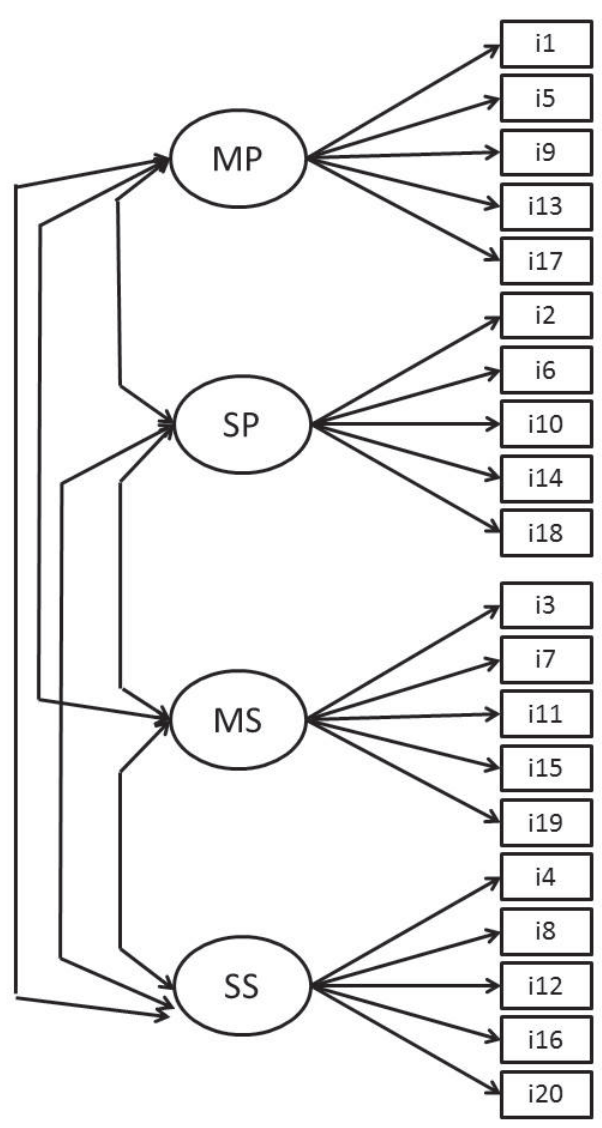

Dans ce modèle, le score de chaque item, représenté par un carré, est fonction linéaire d'une et une seule approche représentée par un cercle; Motivation Profonde (MP), Stratégie Profonde (SP), Motivation de Surface (MS) et Stratégie de Surface (SS). Les doubles flèches représentent les corrélations entre les approches quand elles ne sont pas nulles.

Fig. 1. Modèle complet du questionnaire R-SPQ-2F proposé par Biggs ${ }^{[5]}$.

Variables d'étude

\section{Variable " expliquée »}

La variable « expliquée » est la réussite aux examens partiels qui ont eu lieu le mois suivant l'administration du questionnaire et concernent l'ensemble des inscrits. Nous avons préféré nous intéresser ici à la réussite des étudiants en fin de premier semestre plutôt qu'en fin d'année lors du concours car il existe à Lyon sept concours différents et un étudiant peut s'inscrire à plusieurs concours. Cette situation aurait compliqué l'analyse et l'interprétation des résultats.

Nous avons donc considéré que les $20 \%$ des étudiants les mieux classés au semestre 1 sont ceux qui auront la plus forte probabilité de réussite au concours et nous les avons désignés par la lettre $\mathrm{S}$ comme « Succès potentiel ». Les étudiants moins bien classés ont été désignés par $\mathrm{E}$, comme «Echec potentiel ». Le taux de $20 \%$ choisi correspond au pourcentage de réussite au concours de médecine cette année-là.

\section{Variables « explicatives " d'intérêt}

Nous avons étudié ici l'impact des approches adoptées par les étudiants juste avant les partiels sur leur réussite sans tenir compte des caractéristiques et du cursus passé des étudiants. Les variables « explicatives 》 correspondent donc aux approches d'apprentissage fines mesurées par le R-SPQ-2F dans ce contexte de PACES. Leur mise en évidence est réalisée par l'analyse de la structure factorielle du questionnaire détaillée ci-dessous. Les termes « variable expliquée » et «variables explicatives » sont ici à prendre au sens statistique du modèle car les étudiants adoptent certaines approches à cause de la forme du concours. Il est donc plus juste de parler d'une étude d'association entre les approches et la réussite.

\section{Caractéristiques des étudiants}

Nous disposions pour chaque étudiant de l'âge, du genre et de sa situation universitaire antérieure (série du baccalauréat et mention obtenue, primant/ redoublant en PACES).

\section{Analyse de la structure factorielle du R-SPQ-2F}

\section{Effet plancher ou plafond}

Nous avons étudié l'éventuelle présence d'un effet plancher ou plafond pour chaque item. Un effet plancher (ou plafond) serait observé si une très grande majorité des étudiants cochent la catégorie de réponse la plus faible (ou la plus forte) c'est-à-dire la valeur 1 (réponse A) (ou 5, réponse B). 


\section{Etude du modèle complet du R-SPQ-2F}

\section{Cohérence interne}

Le coefficient alpha de Cronbach, mesure classique de la cohérence interne d'un questionnaire, a été calculé. Il donne le degré avec lequel les items qui mesurent la même dimension (ou sous-dimension) sont inter-corrélés entre eux. Un coefficient de Cronbach supérieur à 0,70 est considéré satisfaisant ${ }^{[15]}$.

\section{Analyse factorielle confirmatoire (AFC)}

Une analyse factorielle confirmatoire permet de juger de la pertinence du modèle complet du R-SPQ2F dans le contexte de la PACES. Les index d'ajustement présentés dans cet article sont le Root Mean Square Error of Approximation (RMSEA) et le Comparative Fit Index $(\mathrm{CFI})^{[16,17]}$. Plus le RMSEA est petit et plus le modèle est bon. Le CFI donne la proportion relative d'amélioration de l'ajustement du modèle proposé par rapport à un modèle où tous les items seraient indépendants. Un CFI de 1 indique un modèle parfait. En pratique, les critères retenus pour apprécier la qualité d'un modèle sont : 1) pour le RMSEA : $\leq 0,06$ bon modèle, entre 0,06 et 0,08 , modèle acceptable, et mauvais modèle au-delà ; 2) pour le CFI $: \geq 0,95$, bon modèle et entre 0,90 et 0,95 , modèle acceptable.

\section{Analyse factorielle exploratoire (AFE) du R-SPQ-2F}

Chaque individu possède 20 coordonnées (ses scores aux 20 items) et peut ainsi être représenté par un point dans un espace à 20 dimensions. L'analyse factorielle exploratoire permet de trouver l'espace de plus petite dimension qui passe au plus près du nuage de points formé par tous les individus. Chaque dimension de ce sous-espace correspond à un facteur sousjacent qui est une variable latente non directement observée. A chaque facteur latent est associée une valeur propre calculée à partir de la matrice de corrélation polychorique des 20 items en tenant compte de leur nature ordinale. Pour limiter l'influence des items asymétriques lors de l'estimation des paramètres, nous avons utilisé l'estimateur pondéré robuste (WLSMV) développé dans MPlus. Nous avons également utilisé une procédure de rotation oblique des facteurs (rotation geomin) permettant aux facteurs d'être corrélés entre eux et assurant l'interprétation la plus pertinente de ces facteurs. Les facteurs qui peuvent suffire à rendre compte de la forme du nuage de points sont ceux qui ont les plus fortes valeurs propres. Ces facteurs peuvent être interprétés comme des variables latentes expliquant la variation des scores observée entre individus. Les facteurs retenus sont ceux pour lesquels cette valeur propre est significativement supérieure à 1 , ce qui a été déterminé en utilisant la méthode de Horn ${ }^{[18]}$.

Nous avons ensuite obtenu les corrélations de chaque item avec les facteurs latents retenus et sélectionné les meilleurs items qui corrélaient le plus ( $|r| \geq$ 0.3 ) avec ces facteurs. Chacun de ces facteurs peut être alors considéré comme une approche d'apprentissage fine et spécifique adoptée par l'étudiant dans le contexte de PACES, dont la signification dépend de la nature des items associés. Chaque item sélectionné doit corréler avec un et un seul facteur si nous souhaitons ensuite utiliser la somme (ou la moyenne) des scores de ces items pour estimer l'approche latente mesurée par ces items. Nous avons donc éliminé les items qui ne corrélaient avec aucun facteur et ceux qui corrélaient avec plus d'un facteur. Le modèle retenu sera validé par une analyse factorielle confirmatoire réalisée dans un contexte de modélisation en équations structurelles.

Association entre les approches et la réussite

\section{Association entre les approches d'apprentissage fines et la bonne performance aux partiels de PACES semestre 1}

Variable « expliquée »

La variable « expliquée » est le Succès potentiel (S/E) en PACES, défini précédemment.

Variables « explicatives » d'intérêt : les approches d'apprentissage

Nous avons calculé pour chaque sujet la moyenne des scores des items associés à chaque facteur latent retenu par l'analyse factorielle exploratoire précédente puisque ces facteurs définissent les approches d'apprentissage fines et spécifiques adoptées dans ce 
contexte de PACES. Afin de faciliter l'interprétation des résultats, nous avons simplifié les scores moyens de chaque approche en donnant la valeur 1 aux étudiants au-dessus de la médiane et la valeur 0 pour les autres.

\section{Analyse multivariée}

Un modèle de régression binomiale avec une fonction de lien logarithme népérien nous a permis d'étudier l'association du taux de succès potentiel $\mathrm{S}$ avec les approches, sans tenir compte du passé académique de l'étudiant car ce passé est lié aussi aux approches qu'a pu adopter l'étudiant dans des contextes antérieurs. Les interactions entre les différentes approches sur le taux de succès ont été évaluées.

\section{Etude des approches adoptées selon le passé acadé- mique de l'éfudiant}

Pour le passé, nous n'avons pris en compte que la mention obtenue au baccalauréat et le redoublement puisque la grande majorité des étudiants avait passé le même type de baccalauréat (baccalauréat scientifique). Pour la mention, nous avons distingué deux groupes : mention forte désignée par $\mathrm{M}^{+}$(Mention Bien ou Très Bien) et mention faible désignée par $\mathrm{M}^{-}$ (mention assez bien ou passable). Nous avons désigné par Pri les étudiants primants n'ayant pas redoublé la PACES, et par Red les étudiants ayant redoublé ou triplé. Après normalisation non paramétrique des scores des approches (scores normaux), nous avons comparé les moyennes des approches entre les quatre groupes $\left(\right.$ PriM $^{-}$, PriM $^{+}$, RedM $^{-}$et RedM ${ }^{+}$) par une analyse de variance (ANOVA), et en cas de significativité par un test post-hoc de TukeySHD. Pour chaque approche, afin d'évaluer la taille de l'effet du groupe lorsque deux moyennes étaient significativement différentes, nous avons standardisé cette différence en la divisant par l'écart type commun aux deux groupe. D'après Cohen ${ }^{[19]}$, un effet est considéré petit pour une différence standard de 0,10 ; modéré pour 0,30 et important pour 0,50 et au-delà. Nous avons également étudié l'effet du genre et du passé académique des étudiants sur leurs approches.

Les analyses statistiques ont été effectuées en langage $\mathrm{R}$ version 3.2.2 et avec le logiciel Mplus version 7.1. Ces logiciels sont accessibles sur leur site web respectif : https://cran.r-project.org/ et https:// www.statmodel.com/. La limite du seuil de signification (risque $\alpha$ de $1^{\circ}$ espèce) a été choisie à 0,05 pour les différentes analyses.

\section{Résultats}

\section{Participants}

Au total, 1977 étudiants sont inclus dans l'analyse. Le diagramme des flux est donné en annexe 5. L'âge moyen est de 18,9 années avec un écart type de 1,3 et 692 étudiantes sur 1977 étudiants, soit 65,0\%, sont des filles. La réponse à chaque item étant nécessaire pour passer à l'item suivant, un seul étudiant a présenté des valeurs manquantes.

\section{Structure factorielle du questionnaire R-SPQ-2F}

\section{Effet plancher ou plafond}

Aucun effet plancher ou plafond n'est observé puisque pour chaque item, aucune des deux catégories de réponse extrêmes (valeur 1 ou 5) n'est sélectionnée avec une fréquence de plus de $75 \%$.

\section{Etude du modèle complet du R-SPQ-2F}

\section{Cohérence interne (tableau I)}

Les valeurs du coefficient de Cronbach sont peu acceptables surtout pour les stratégies et la motivation de surface, pour lesquelles les valeurs sont très inférieures à 0,7 (entre 0,47 et 0,49 ). Ceci suggère un manque de corrélation entre ces items de même nature supposés mesurer la même dimension ou sous-dimension.

\section{Analyse factorielle confirmatoire (AFC)}

Une analyse factorielle confirmatoire avec le modèle complet du R-SPQ-2F représenté figure 1 nous indique un mauvais modèle selon les critères adoptés (RMSEA $=0,084$, CFI de 0,74). Il nous a alors fallu procéder à une analyse factorielle exploratoire. 
Tableau I. Coefficients alpha de Cronbach pour les dimensions et sous-dimensions du R-SQP-2F définies par Biggs dans le cadre de la première année commune des études de santé.

\begin{tabular}{|l|c|c|c|}
\hline & Effectif & Nombre d'items & alpha \\
\hline Approche Profonde (AP) & 1977 & 10 & 0,70 \\
\hline Approche de Surface (AS) & 1977 & 10 & 0,64 \\
\hline Motivation Profonde (MP) & 1977 & 5 & 0,61 \\
\hline Stratégie Profonde (SP) & 1977 & 5 & 0,47 \\
\hline Motivation de Surface (MS) & 1977 & 5 & 0,49 \\
\hline Stratégie de Surface (SS) & 1977 & 5 & 0,48 \\
\hline
\end{tabular}

Tableau II. Valeurs propres et pourcentage de variabilité des données expliquées par les cinq premiers facteurs obtenus lors de l'analyse factorielle exploratoire de la version française du R-SPQ-2F à 20 items de Biggs dans le cadre de la première année commune des études de santé.

\begin{tabular}{|l|c|c|c|c|c|}
\hline & Facteur 1 & Facteur 2 & Facteur 3 & Facteur 4 & Facteur 5 \\
\hline Valeur propre & 3,79 & 2,19 & 1,91 & 1,33 & 1,06 \\
\hline$\%$ expliqué par facteur & $19 \%$ & $11 \%$ & $10 \%$ & $7 \%$ & $5 \%$ \\
\hline \% cumulé & $19 \%$ & $30 \%$ & $39 \%$ & $46 \%$ & $51 \%$ \\
\hline
\end{tabular}

\section{Analyse factorielle exploratoire (AFE) du R-SPQ-2F}

\section{Facteurs latents mis en évidence}

Les quatre premiers facteurs expliquent $46 \%$ de la variabilité totale des données observées (tableau II). Pour les données générées au hasard sans structure factorielle, l'analyse de Horn donne un quantile $95 \%$ de 1,10 pour la valeur propre du cinquième facteur. La valeur propre estimée du facteur 5 avec nos données est égale à 1,06 et est donc inférieure à cette valeur seuil de 1,10 . Ainsi, le cinquième facteur n'est pas retenu comme facteur pertinent et le R-SQP-2F a une structure à quatre facteurs qu'il faut interpréter. Le tableau III donne pour chaque item sa corrélation avec les facteurs latents retenus lorsqu'elle est supérieure à 0,3 en valeur absolue.

\section{Sélection des items et interprétation des facteurs}

Nous avons éliminé les items qui corrèlent peu $(|r|<$ 0,3 ) avec les quatre facteurs ainsi que ceux qui corrèlent avec plus d'un facteur afin de pouvoir utiliser la moyenne des scores pour chaque sous-dimension.

Deux items corrèlent peu avec les quatre facteurs retenus (i17, i18). Quatre items corrèlent avec au moins deux facteurs retenus (i6, i14, i19 et i16) et l'item 15 corrèle à la fois peu et avec deux facteurs
$(0,27$ avec le facteur 2 et 0,24 avec le facteur 3$)$. En éliminant ces 7 items, nous avons obtenu un questionnaire à 13 items et 4 facteurs.

Le tableau IV résume les approches fines que l'on peut mettre en évidence dans le cadre de la PACES. Nous avons un premier facteur relatif à l'approche profonde (AP) car il corrèle à la fois avec quatre items de la motivation profonde et deux items de la stratégie profonde, et trois facteurs relatifs à l'approche de surface. Le deuxième facteur relève plutôt de la motivation de surface : réussite au moindre coût intellectuel que l'on appellera Réussite la plus Efficace (RE), les facteurs 3 et 4 concernent deux stratégies de surface : le ciblage de l'information donnée en cours que l'on appellera Focalisation sur le Cours (FC) (items 4 et 12) et la Mémorisation par Coeur (MpC) (items 11, 8 et 20).

L'analyse factorielle confirmatoire effectuée sur notre questionnaire PACES à 13 items présente un ajustement très acceptable $($ RMSEA $=0,049$ et $\mathrm{CFI}=$ 0,943 pour l'ensemble de la cohorte de PACES. Les coefficients standardisés du modèle sont donnés figure 2. L' « approche profonde » corrèle négativement avec la RE $\left(r=-0,55 ; p<10^{-3}\right)$. L'approche $\mathrm{MpC}$ corrèle positivement avec la RE et avec la FC $\left(0,19\right.$ et 0,23 respectivement ; $\left.p<10^{-3}\right)$. L' « approche profonde » est indépendante de l'approche FC et 
Tableau III. Résultats de l'analyse factorielle exploratoire de la version française du R-SPQ-2F à 20 items en première année commune des études de santé.

\begin{tabular}{|c|c|c|c|c|c|c|}
\hline \multirow{2}{*}{\multicolumn{2}{|c|}{$\begin{array}{l}\text { Dimensions et } \\
\text { Sous dimensions } \\
\text { définies par Biggs }\end{array}$}} & $\mathrm{n}^{\circ}$ & \multicolumn{4}{|c|}{ Facteurs latents retenus } \\
\hline & & item & 1 & 2 & 3 & 4 \\
\hline \multirow{10}{*}{ 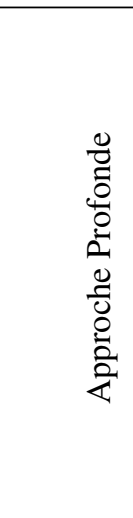 } & \multirow{5}{*}{ 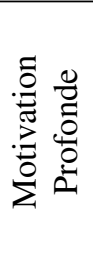 } & 1 & $\mathbf{0 , 5 9}$ & & & \\
\hline & & 5 & $\mathbf{0 , 5 2}$ & & & \\
\hline & & 9 & 0,61 & & & \\
\hline & & 13 & 0,65 & & & \\
\hline & & 17 & & & & \\
\hline & \multirow{5}{*}{ 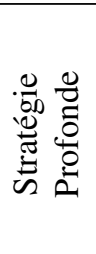 } & 2 & 0,43 & & & \\
\hline & & 6 & 0,46 & & $-0,45$ & \\
\hline & & 10 & 0,43 & & & \\
\hline & & 14 & 0,39 & & $-0,48$ & \\
\hline & & 18 & & & & \\
\hline \multirow{10}{*}{ 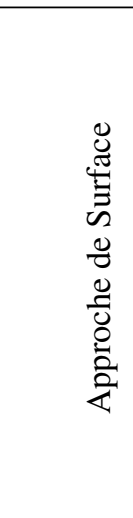 } & \multirow{5}{*}{ 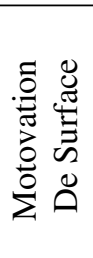 } & 3 & & 0,66 & & \\
\hline & & 7 & & 0,67 & & \\
\hline & & 11 & & & & 0,69 \\
\hline & & 15 & & & & \\
\hline & & 19 & & 0,46 & 0,39 & \\
\hline & \multirow{5}{*}{ 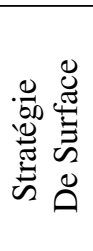 } & 4 & & & 0,65 & \\
\hline & & 8 & & & & 0,56 \\
\hline & & 12 & & & 0,62 & \\
\hline & & 16 & & 0,48 & 0,45 & \\
\hline & & 20 & & & & $\mathbf{0 , 3 0}$ \\
\hline
\end{tabular}

Les 20 items du R-SPQ-2F sont numérotés selon leur ordre de passation et triés par type d'approche (profonde et de surface) puis par sous dimensions : motivation et stratégie. Les corrélations de chaque item avec les différents facteurs latents retenus sont données si elles sont supérieures à 0,3 en valeur absolue. Les items en gras correspondent aux items conservés et les items de graisse normale aux items supprimés soit parce qu'ils ne corrèlent suffisamment avec aucun facteur (numéro des items surligné en gris), soit parce qu'ils corrèlent avec plus d'un facteur (ligne des items surlignée en gris).

corrèle négativement peu avec l'approche $\mathrm{MpC}(r=$ $-0,07 ; p=0,02)$.

\section{Association entre les approches et la réussite}

\section{Association entre les approches d'apprentissage fines adoptées et le succès potentiel en PACES (figure 3)}

Les effets des approches d'apprentissage sur le succès potentiel des étudiants sont additifs.
Les étudiants avec forte approche profonde ont plus de chances de succès potentiel que les étudiants avec moins d'approche profonde $\left(\mathrm{RR}_{95 \%}=1,5\right.$; $\left.\mathrm{IC}_{95 \%}=\right] 1,3 ; 1,9\left[, p<10^{-5}\right)$. Les étudiants apprenant par cœur ont moins de chances de succès potentiel que ceux qui apprennent moins par cœur $\left(\mathrm{RR}_{95 \%}=\right.$ 0,$\left.62 ; \mathrm{IC}_{95 \%}=\right] 0,52 ; 0,74\left[, p<10^{-7}\right)$, ce qui revient à dire qu'ils ont 1,6 plus de risque d'échouer. Les étudiants se focalisant sur l'information donnée en cours ont 1,4 plus de chances de succès potentiel que ceux qui ciblent moins l'information $\left(\mathrm{RR}_{95 \%}=\right] 1,2 ; 1,6[$, 
Tableau IV. Approches fines mises en évidence dans le cadre de la première année commune des études de santé à partir de l'analyse factorielle exploratoire du R-SQP-2F à 20 items.

\begin{tabular}{|l|l|c|c|c|}
\cline { 2 - 5 } \multicolumn{1}{c|}{} & \multicolumn{1}{c|}{ PACES } & items & \multicolumn{2}{c|}{ R-SPQ-2F } \\
\cline { 2 - 5 } \multicolumn{1}{c|}{} & \multicolumn{1}{c|}{ Approches fines } & & Sous-Dimension & Dimension \\
\hline Facteur 1 & Approche Profonde (AP) & $\begin{array}{c}\mathrm{i} 1, \mathrm{i} 5, \mathrm{i} 9, \\
\mathrm{i} 13, \mathrm{i} 2, \mathrm{i} 10\end{array}$ & $\begin{array}{c}\text { Motivation } \\
\text { et stratégie Profonde }\end{array}$ & $\begin{array}{c}\text { Approche } \\
\text { Profonde }\end{array}$ \\
\hline Facteur 2 & Réussite la plus Efficace (RE) & $\mathrm{i} 3, \mathrm{i} 7$ & Motivation de Surface & Approche de \\
Surface
\end{tabular}

$\left.p<10^{-3}\right)$. La RE a un effet presque significatif sur le succès potentiel $\left(\mathrm{RR}=1,2, \mathrm{RR}_{95 \%}=\right] 1,0 ; 1,4[, p=$ $0,07)$.

\section{Etude des approches adoptées selon le passé académique de l'étudiant (figure 4)}

Les moyennes de chaque approche fine pour les quatre groupes: PriM ${ }^{-}$, PriM $^{+}$, RedM $^{-}$et RedM ${ }^{+}$sont représentées figure 4. Les ANOVAs réalisées (une par approche) sont significatives. Dans ces modèles, l'approche correspond à la variable « expliquée » et la variable " groupe » à la variable « explicative». Seul le groupe PriM a présenté des différences significatives avec au moins un autre groupe pour toutes les approches. Ce groupe particulier des primants à faible mention au baccalauréat fait preuve d'une approche profonde faible et d'approches de surface fortes par rapport aux trois autres groupes. Pour les approches «Approche Profonde », « Réussite la plus Efficace » et «Focalisation sur le Cours », la taille des effets les plus forts est de l'ordre de 0,20 en valeur absolue ce qui correspond à des effets à mi-chemin entre effet faible et effet modéré. Par contre pour l'approche « Mémorisation par Cœur », l'ampleur de l'effet groupe $(0,28)$ est modérée $(\sim 0,30)$. Ce groupe PriM $^{-}$est numériquement très important puisqu'il représente presque la moitié des étudiants analysés soit $42 \%$ (831/1977). Nous avons obtenu les mêmes observations sur les étudiants n'ayant passé que des baccalauréats scientifiques (représentant $95 \%$ de la cohorte).
Le tableau V donne le pourcentage d'étudiants avec succès potentiel pour les quatre groupes d'étudiants $\left(\right.$ PriM $^{-}$, PriM $^{+}$, RedM ${ }^{-}$et $\left.\operatorname{RedM}^{+}\right)$. Nous constatons le faible pourcentage de succès potentiel pour les étudiants primants sans mention forte $(2,1 \%)$ et l'effet positif du redoublement sur le succès potentiel qui est 12 fois plus fort en pourcentage dans le cas des mentions faibles et qui double dans le cas des mentions fortes.

En tenant compte des deux derniers points abordés, approches d'apprentissage et succès potentiel selon les groupes, nous constatons que les étudiants primants avec mention faible ont des approches d'apprentissage très différentes des autres étudiants et réussissent beaucoup moins bien $(2,1 \%$ versus $33 \%$ (382/1143)).

\section{Effet du genre}

Le pourcentage de succès potentiel chez les garçons est de 1,4 fois plus fort que chez les filles (intervalle de confiance $\left.\mathrm{RR}_{95 \%}=\right] 1,1 ; 1,7[$ ).

L'analyse de la variance de chaque approche en fonction du passé et du genre, pris dans un seul modèle, nous indique que l'effet du genre sur l'approche est similaire dans les différents groupes $\left(\right.$ PriM$^{-}$, PriM $^{+}$, RedM $^{-}$et $\operatorname{RedM}^{+}$) et ceci pour les quatre approches. Les garçons diffèrent significativement des filles seulement pour l'approche « Réussite la plus Efficace », prépondérante chez les garçons $\left(p<10^{-15}\right)$. Cette différence de moyennes de $\operatorname{RE}(0,3)$ entre fille et garçons est deux à trois fois plus 


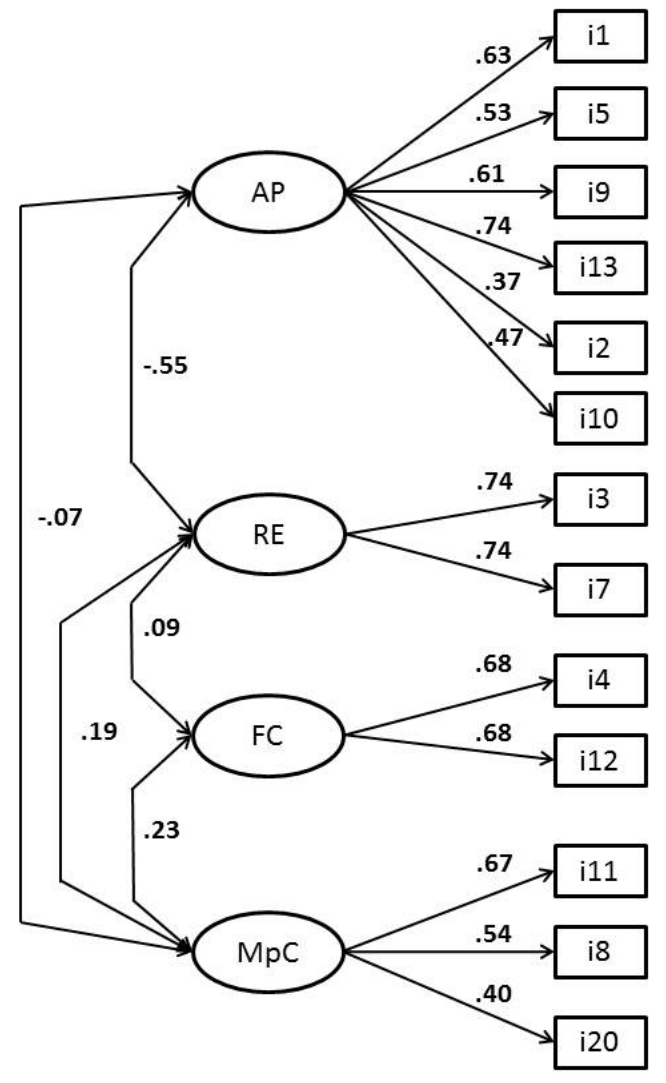

Les quatre approches d'apprentissage fines (cercles) mises en évidence en PACES sont: l'Approche Profonde (AP), la Réussite la plus Efficace (RE), la Focalisation sur le cours $(F C)$ et la Mémorisation par Cour $(M p C)$. Les corrélations entre le score continu sous-jacent de chaque item (carré) et son approche sont indiquées sur les flèches unidirectionnelles. Les flèches bidirectionnelles donnent les corrélations entre les approches prises 2 à 2 lorsqu'elles ne sont pas nulles.

Fig. 2. Analyse factorielle confirmatoire du questionnaire première année commune des études de santé (PACES) à 13 items mis en évidence à partir de la version française du RSPQ-2F.

importante pour les groupes à faible mention (PriM ${ }^{-}$ et $\operatorname{RedM}^{-}$) que pour les deux autres groupes.

\section{Discussion}

Grâce à l'analyse factorielle du R-SPQ-2F de Biggs $^{[5]}$, nous avons pu retenir quatre approches d'apprentissage fines qui correspondent au mieux au contexte de la PACES à Lyon. Nous avons alors pu montrer que l'approche profonde des étudiants et la focalisation sur le contenu du cours permettent une meilleure réussite des étudiants aux partiels du semestre 1 de PACES contrairement à la mémorisation par cœur.

\section{Structure factorielle du R-SQP-2F en PACES}

Nous ne retrouvons pas quatre facteurs identiques à ceux de Biggs ${ }^{[5]}$. Ceci confirme que ce questionnaire est très sensible au contexte pédagogique et culturel $^{[6,7,20]}$. Cependant, après sélection des items, certains auteurs arrivent à mettre en évidence quatre facteurs dont deux sont relatifs à l'approche profonde et deux relatifs à l'approche de surface ${ }^{[7-9]}$. Le plus frappant dans notre contexte de PACES est qu'après sélection des items, nous ne trouvons qu'un seul facteur relatif à l' approche profonde comprenant surtout des items de motivation profonde et trois facteurs relatifs à la surface qui sont très prononcés. Ces résultats différents de la littérature peuvent être liés au caractère très spécifique et sélectif du concours de PACES.

\section{Items éliminés}

\section{Items peu corrélés avec les quatre facteurs retenus}

Les deux items 17 et 18 correspondent peu à la réalité d'un étudiant préparant la PACES. Ainsi, l'item 17 (J'arrive aux cours avec en tête des questions pour lesquelles j'aimerais des réponses) n'est guère pertinent compte-tenu du taux d'encadrement extrêmement faible en PACES (un enseignant pour plus de 1000 étudiants). Ceci ne permet pas à l'enseignant de répondre aux questions personnelles de chaque étudiant. Cet item 17 est également éliminé par d'autres auteurs pour ses faibles corrélations, dans le cas d'une population d'étudiants d'une université belge provenant de différentes disciplines et concernant toutes les années d'étude ${ }^{[9]}$.

Pour l'item 18 (Je m'assure d'avoir regardé la plupart des lectures conseillées qui accompagnent les cours), les enseignants conseillent rarement des lectures pour approfondir les notions vues en cours. En 


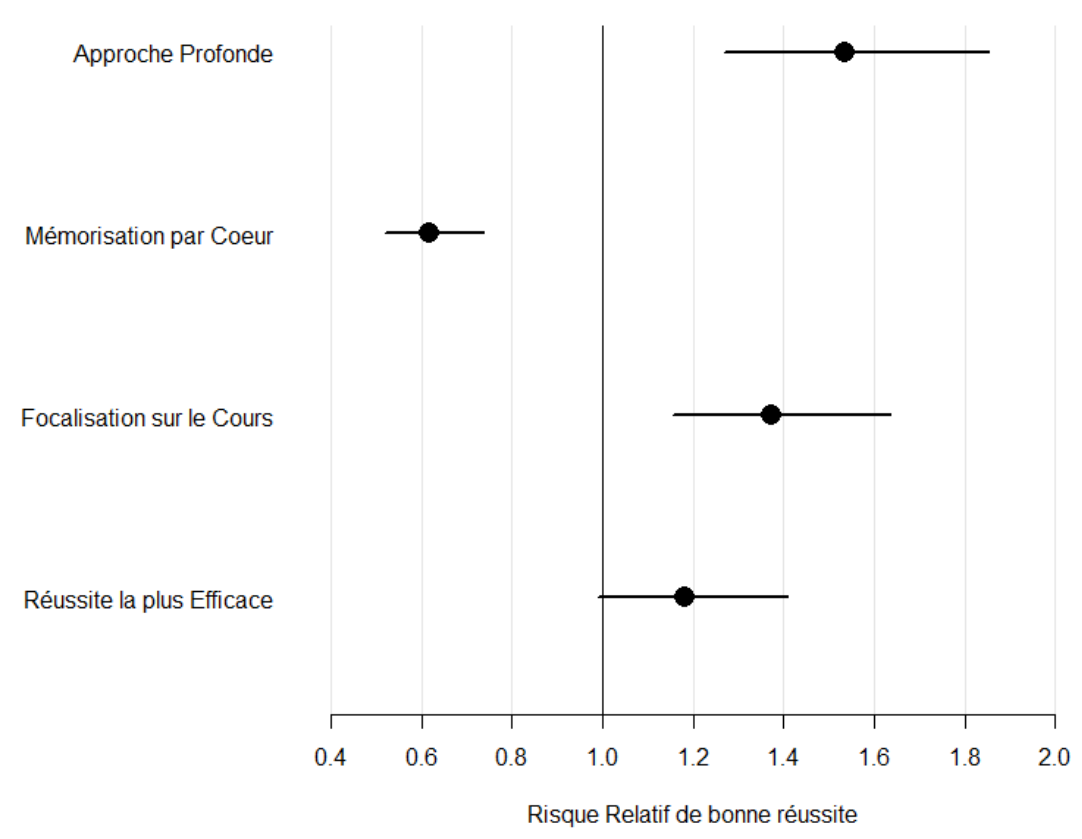

Les risques relatifs de succès potentiel en PACES $1^{\circ}$ semestre sont représentés par des points avec leur intervalle de confiance à $95 \%$ respectif pour les quatre approches. Le risque relatif pour les différentes approches d'apprentissage correspond au facteur multiplicatif permettant de passer de la probabilité de Succès potentiel chez la moitié des étudiants avec un score le plus faible pour l'approche à la probabilité de Succès potentiel chez les autres étudiants au score plus élevé. Un étudiant est considéré avoir un succès potentiel en PACES s'il est classé parmi les $20 \%$ meilleurs aux examens partiels de décembre du concours de PACES.

Fig. 3. Risque relatif de succès potentiel aux partiels de première année commune des études de santé (PACES) en fonction des différentes approches d'apprentissage fines adoptées par les étudiants en PACES.

effet, pour garantir une égalité pour tous et éviter les recours juridiques, ils interrogent uniquement sur les informations données en cours. Il est même souvent conseillé aux étudiants de travailler principalement sur les annales et d'apprendre à répondre aux questions à choix multiples (QCM).

\section{Items corrélés avec au moins deux facteurs retenus}

Certains items sont corrélés avec deux approches fines en positif ou négatif. Ces items sont éliminés du fait de leur manque de spécificité vis-à-vis des approches. La formulation de ces items évoque deux approches voisines ou opposées qui sont séparées dans le modèle, ce qui fait apparaitre des corrélations entre les deux approches.
Nous constatons que nous avons éliminé plus de la moitié des items de la stratégie profonde du R-SQP$2 \mathrm{~F}$ original. Cette stratégie profonde consiste à élargir le domaine des connaissances par un travail personnel d'autoformation.

\section{Signification des approches retenues}

L'approche profonde de notre modèle comprend plutôt des items qui touchent l'intérêt intrinsèque aux études et des items de stratégies profondes (i2, i10) qui peuvent s'interpréter dans ce contexte comme une activité de « bachotage ». L'intérêt porté aux études facilite le travail de bachotage.

L'approche « Réussite la plus Efficace » $(\mathrm{i} 3, \mathrm{i} 7)$ a une faible moyenne de score et concerne peu 
Approche Profonde (AP)

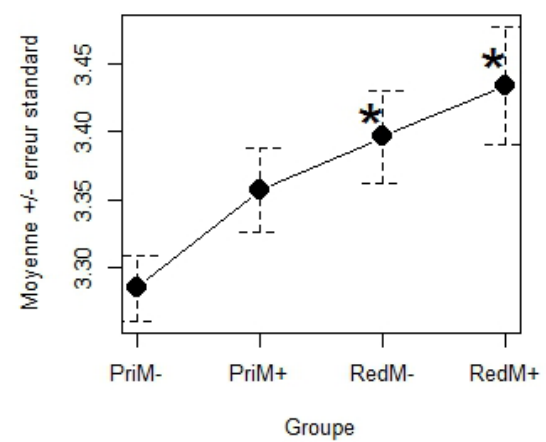

Focalisation sur le Cours (FC)

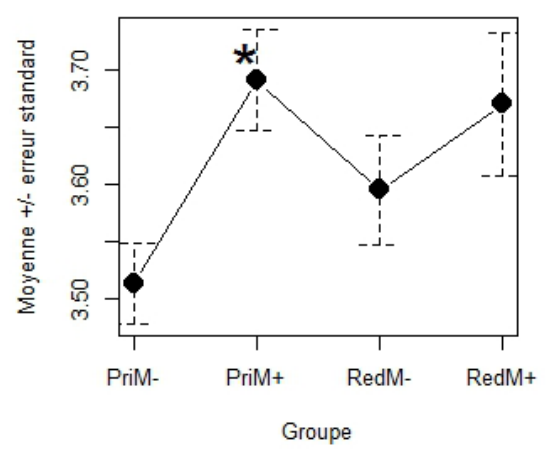

Réussite la plus Efficace (RE)

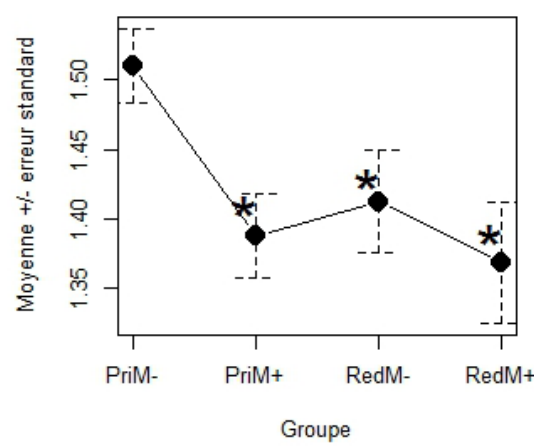

Mémorisation par Coeur (MpC)

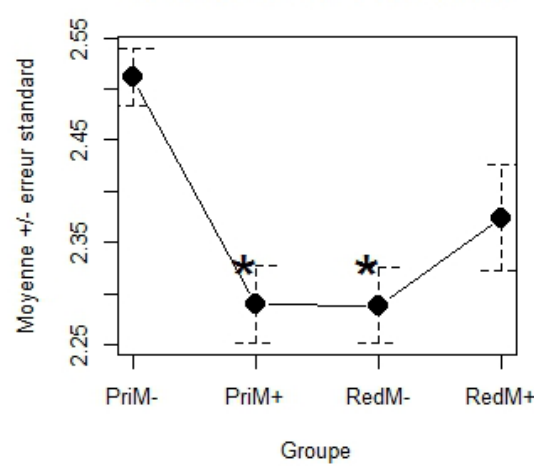

Les seules différences significatives obtenues 2 à 2 par un test post-hoc TukeyHSD sont indiquées par une étoile. Elles n'ont été obtenues qu'entre le $1^{\circ}$ groupe (primants à faible mention au baccalauréat ; PriM-) et les autres groupes marqués d'une étoile (Primants à forte mention : PriM ${ }^{+}$; redoublant à faible mention : RedM ou redoublant à forte mention : $\left.\operatorname{Red} M^{+}\right)$.

Fig. 4. Approches d'apprentissage fines adoptées en première année commune des études de santé (PACES) en fonction des antécédents universitaires (redoublement et mention au baccalauréat).

d'étudiants. Ces deux items sont éliminés chez deux auteurs ${ }^{[7,9]}$ et au moins un des deux items est éliminé chez deux autres auteurs ${ }^{[8,20]}$ du fait de leur corrélation avec deux facteurs à la fois dans leur contexte.

L'approche «Focalisation sur le Cours » correspond aux consignes plus ou moins explicites données en PACES comme évoqué précédemment.

La mémorisation par cœur sans forcément comprendre relève de la mémorisation pure en adéquation avec une évaluation par QCM factuelles.

Le concours de PACES, tel qu'il est perçu par les étudiants ou expliqué par les enseignants et/ou les tuteurs, devient la cause des approches adoptées.
Mais, par ailleurs, les approches adoptées font partie des causes des résultats aux concours puisqu'elles le précèdent. Ainsi, nous avons un système en boucle pour lequel il est difficile de distinguer les causes des effets. Il est nécessaire d'analyser de quelle façon les étudiants sont évalués pour comprendre les approches d'apprentissage qu'ils adoptent.

\section{Etude des chances de réussir en fonction des approches d'apprentissage}

Une plus grande approche en profondeur est en faveur de la réussite. En effet, pour fournir autant de 
Tableau V. Succès potentiel en première année commune des études de santé (PACES) selon le redoublement et la mention au baccalauréat des étudiants.

\begin{tabular}{|l|c|c|c|}
\hline & Succès potentiel & $\begin{array}{c}\text { Echec } \\
\text { potentiel }\end{array}$ & Total \\
\hline & $n(\%)$ & $n(\%)$ & $n(\%)$ \\
\hline Primant mention faible $($ PriM & & $827(42 \%)$ \\
\hline Primant mention forte $\left(\right.$ PriM $\left.^{+}\right)$ & $17(2,1 \%)$ & $810(98 \%)$ & $473(24 \%)$ \\
\hline Redoublant mention faible $\left(\mathrm{RedM}^{-}\right)$ & $132(28 \%)$ & $341(72 \%)$ & $423(21 \%)$ \\
\hline Redoublant mention forte $\left(\mathrm{RedM}^{+}\right)$ & $105(25 \%)$ & $318(75 \%)$ & $247(13 \%)$ \\
\hline
\end{tabular}

Un étudiant est considéré avoir un succès potentiel en PACES s’il est classé parmi les $20 \%$ meilleurs aux examens partiels de décembre du concours de PACES.

travail qu'il est demandé en PACES en moins de quatre mois, il est nécessaire d'aimer et de s'intéresser à ce que l'on apprend. Certains auteurs ont montré qu'une approche profonde favorisait la réussite en première année d'études médicales ou de sciences alors qu'une approche de surface était en défaveur $^{[21]}$.

C'est sur l'approche de surface que nos résultats sont divergents. En effet, en PACES, une forte focalisation sur le contenu du cours (stratégie de surface) est en faveur de la réussite parce que les enseignants n'interrogent que sur le contenu de leurs cours magistraux pour des questions plus « juridiques » que pédagogiques. Par ailleurs, une forte mémorisation par cœur (stratégie de surface) est en faveur de l'échec. La quantité à retenir est si importante que les étudiants doivent structurer leur mémoire ce qui est facilité par la compréhension de ce qu'ils apprennent et un certain effort de synthèse.

Par ailleurs, nos résultats confirment : 1) que les étudiants avec mention au baccalauréat ont plus de chance de réussir dans des cursus de santé en France $^{[22]}$ et à l'étranger ${ }^{[23]}$ et 2) que les redoublants réussissent mieux que les primants ${ }^{[24]}$.

Contrairement aux examens traditionnels ${ }^{[25]}$, dans le cadre de la PACES, les garçons réussissent mieux que les filles. Dans notre population, les filles avaient totalisé un score au stress plus fort que les garçons lors de l'administration d'un questionnaire élaboré par nos soins et relatif à leur stress vis-à-vis de leur travail en PACES (score de 8,7/16 vs. 7,2/16 pour respectivement les filles et les garçons). Les filles pourraient moins bien réussir que les garçons en partie à cause de leur stress plus important ${ }^{[26]}$.

\section{Analyse des approches d'apprentissage adoptées en fonction du passé académique des étudiants puis du genre}

Grâce à ces approches fines qui ne correspondent pas tout à fait aux facteurs de la version originale du R-SQP-2F, nous avons pu mettre en évidence une liaison entre le passé académique des étudiants et leurs approches. En effet, les étudiants primants à faible mention au baccalauréat ont la plus faible approche en profondeur (moins d'intérêt pour leurs études et moins de travail personnel) et la plus forte motivation de surface, dans la mesure où ils ont plus souvent pour objectif de réussir le plus efficacement possible.

Les étudiants redoublants à faible mention présentent un comportement d'apprentissage voisin des étudiants avec forte mention (primant ou redoublant). Ceci peut être expliqué par l'effet de sélection et le changement d'approches d'apprentissage. En effet, environ un quart des étudiants primants avec mention faible qui échouent en médecine ne se réinscrivent pas une seconde fois en PACES et ceux qui redoublent apprendraient à adopter des approches plus conformes au contexte de leurs études. Ceci confirme que les approches d'apprentissage dépendent du contexte des études ${ }^{[12,13]}$.

Nous notons également une plus forte motivation de surface chez les garçons que chez les filles, ce qui 
correspond à l'idée largement admise dans l'enseignement secondaire : les filles approfondissent plus leur travail scolaire que les garçons d'une façon générale. Dans le contexte de la PACES, elles « perdraient » ainsi du temps à approfondir ce qui pourrait contribuer à leur moins bonne réussite que celle des garçons.

Ces résultats confirment les associations précédentes entre réussite et approches, et entre réussite et passé académique ou genre.

En résumé, pour réussir cette année de PACES, les étudiants doivent avoir adopté une stratégie de surface marquée par l'absence d'approfondissement personnel des notions vues en cours et une exigence de compréhension liée à la quantité de détails à retenir du fait de l'évaluation factuelle. Ceci suggère un apprentissage court terme et un manque d'entraînement à l'auto-formation. Cela met bien en évidence les défauts pédagogiques de la PACES : enseignement de masse centré sur l'enseignant (cours magistraux), mode de sélection essentiellement par QCM factuelles qui est aggravé ici par le caractère très fortement sélectif du concours de PACES. Cette année très difficile à vivre peut avoir également comme effet négatif de décourager les bons étudiants puisqu'environ $10 \%$ des étudiants primants à forte mention échouant au concours de médecine ne se réinscrivent pas en PACES l'année suivante.

Les limites de cette étude proviennent du fait que les données n'ont été collectées que dans une seule université et que les étudiants redoublants et primants n'étaient pas appariés sur deux années consécutives. Par ailleurs, lors de la traduction du questionnaire original de Biggs, nous avons cherché à rester au plus près du texte d'origine, car cette version francophone devait être également utilisée à la faculté de médecine de Genève, donc dans un autre contexte culturel francophone. Ainsi i) aucune adaptation n'a été entreprise en fonction de la culture française, ni en fonction du contexte spécifique des études en France, ou du contexte encore plus spécifique de la PACES et ii) aucun remaniement en profondeur des items n'a été opéré pour les rendre univoque lorsqu'ils véhiculaient deux idées ${ }^{[27]}$. Ce questionnaire gagnerait à être repris en profondeur et mieux adapté à la culture française en suivant des procédures adaptées ${ }^{[28]}$.

\section{Perspectives}

Ce concours très sélectif et très stressant a pour effet d'encourager les étudiants à délaisser certaines stratégies d'apprentissage profondes et de renforcer en partie l'approche de surface. Cette première année pourrait potentiellement diminuer la curiosité des étudiants et leur ouverture d'esprit. L'objectif d'un prochain travail est donc d'évaluer si ces approches perdurent au cours des études de santé.

\section{Contributions}

Marie-Paule Gustin et Siara Isaac ont conçu le protocole de recherche et recueilli les données. Marie-Paule Gustin a effectué l'analyse statistique, interprété les résultats et écrit le manuscrit. Christine Vinciguerra, Carole Burillon et Jérôme Etienne ont permis l'administration des questionnaires puis participé activement à la discussion de cet article.

\section{Remerciements}

Nous remercions : Anna Baroffio de l'Unité de Développement et de Recherche en Education Médicale de l'Université de Genève pour sa relecture attentive et ses conseils avisés tout au long de ce travail ; Karen Spruyt, psychologue statisticienne spécialisée dans le sommeil chez l'enfant, pour sa relecture et sa précieuse expérience sur la conception des questionnaires ; Catherine Cerutti, ingénieure au CNRS et à l'Ecole Normale Supérieure de Lyon pour sa relecture de l'ensemble du travail ; et le Professeur Jean-François Guerin pour son soutien lors de la mise en place de l'étude. Nous remercions également tous les étudiants de la promotion PACES 2011-2012 de l'Université Claude Bernard Lyon 1 qui ont fait preuve de générosité en prenant le temps de répondre malgré la grosse charge de travail qui leur incombait.

\section{Déclaration d'intérêt}

Aucun auteur ne déclare de conflit d'intérêt en lien avec le contenu de cet article 


\section{Approbation éthique}

Le comité d'éthique du centre hospitalo-universitaire de Lyon a donné son accord pour cette étude.

\section{Références}

1. Marton F, Säljö R. On qualitative differences in learning - I: Outcome and process. Br J Educ Psychol 1976;46:4-11.

2. Marton F, Säljö R. On qualitative differences in learning - II: Outcome as a function of the learner's conception of the task. Educ Psychol 1976;46:115127.

3. Tait H, Entwistle NJ, McCune, V. ASSIST: a reconceptualisation of the Approaches to Studying Inventory. In C. Rust (ed.) Improving students as learners. Oxford: Oxford Brookes University, The Oxford Centre for Staff and Learning Development, 1998.

4. Biggs J. Individual and group differences in study processes. Br J Educ Psychol 1978;48:266-279.

5. Biggs J, Kember D, et Leung DYP. The revised twofactor Study Process Questionnaire: R-SPQ-2F. Br J Educ Psychol 2001;71:133-49.

6. Justicia F, Pichardo MC, Cano F, Berbén ABG, De la Fuente J. The Revised Two-factor Study Process Questionnaire (R-SPQ-2F): Exploratory and confirmatory factor analyses at item level. European Journal of Psychology of Education 2008;3:355-72.

7. Immekus JC, Imbrie PK. A test and cross-validation of the Revised Two-factor Study Process Questionnaire factor structure among Western university students. Educ Psychol Meas 2010;70:495-510.

8. Fryer L, Ginns P, Walker R, Nakao K. The adaptation and validation of the CEQ and the R-SPQ-2F to the Japanese tertiary environment. Br J Educ Psychol 2012;82:549-63.

9. Stes A, De Maeyer S, Van Petegem P. Examining the cross-cultural sensitivity of the Revised Two-factor Study Process Questionnaire (R-SPQ-2F) and validation of a Dutch version. PLoS One 2013,8:1-7.

10. Côté DJ, Graillon A, Waddell G, Lison C, Noel MF. L'approche d'apprentissage dans un curriculum médical préclinique basé sur l'apprentissage par problèmes. Pédagogie Médicale 2006;7:201-12.

11. Pottier P, Derkinderen P, Barrier J, Sevin M, Rogez JM, Planchon B. Etude descriptive des pratiques d'apprentissage en quatrième année de médecine dans une faculté française et de leurs performances à résoudre un problème. Pédagogie médicale 2008;9:70-82.

12. Hadwin AF, Winne PH, Stockley DB, Nesbit JC, Woszczyna C. Context moderates students' selfreports about how they study. J Educ Psychol 2001;93:477-87.

13. Kember D, Biggs J, Leung DYP. Examining the multidimensionality of approaches to learning through the development of a revised version of the Learning Process Questionnaire. Br J Educ Psychol 2004;74: 261-80.

14. Leung DYP, Ginns P, Kember D. Examining the cultural specificity of approaches to learning in universities in Hong Kong and Sydney. J Cross Cult Psychol 2008;39:251-266.

15. AERA, APA, NCME. Standards for educational and psychological testing. Washington, DC: American Educational Research Association, 1999.

16. Mac Callum RC, Browne HW, Sugawara HM. Power analysis and determination of sample size for covariance structure modeling. Psychol Methods 1996; 1:130-49.

17. Hu L, Bentler PM. Cutoff criteria for fit indexes in covariance structure analysis: Conventional criteria versus new alternatives. Struct Equ Modeling 1999;6:1-55.

18. Horn JL. A rationale and test for the number of factors in factor analysis. Psychometrika 1965;30:179-85.

19. Cohen J. Statistical power analysis for the behavioral sciences. Hillsdale, NJ: Lawrence Erlbaum, 1987.

20. Socha A, Sigler EA. Exploring and "reconciling" the factor structure for the Revised Two-factor Study Process Questionnaire. Learn Individ Differ 2014; 31:43-50.

21. Salamonson Y, Weaver R, Chang S, Koch J, Bhathal $\mathrm{R}$, Khoo C, et al. Learning approaches as predictors of academic performance in first year health and science students. Nurse Educ Today 2013;33: 729-33.

22. Kohler C, Braun M, Mari G, Roland J. Evolution du profil des étudiants ayant passé le concours de 
PCEM1 à la Faculté de Médecine de Nancy de 1992 à 2001. Pédagogie Médicale 2003;4:12-17.

23. Ferguson E, James D, Madeley L. Factors associated with success in medical school: systematic review of the literature. BMJ 2002;324:952-7.

24. Debouzie D. Rapport du 20 juillet 2003 de la Commission pédagogique nationale de la première année des études de santé, A l'attention de Monsieur le Ministre Luc Ferry, Ministre de la jeunesse, de l'éducation nationale et de la recherche, et de Monsieur le Ministre Jean-François Mattei, Ministre de la santé, de la famille, et des personnes handicapées. France, 2003 [On-line] Disponible sur : http://www.ladocumentationfrancaise.fr/var/storage/rapports-publics/ 034000580.pdf

25. Ministère de l'éducation nationale (Direction de l'évaluation, de la prospective et de la performance). Filles et garçons de l'école à l'enseignement supérieur. 2014 [On-line] Disponible sur : http://cache.media.education.gouv.fr/file/2014/08/ 3/FetG_2014_305083.pdf.

26. Elias H, Ping WS, Abdullah MC. Stress and Academic Achievement among Undergraduate Students in Universiti Putra Malaysia. Procedia - Social and Behavioral Sciences 2011;49:646-55.

27. Streiner DL, Norman GR. Health measurement scales: a practical guide to their development and use. New-York : Oxford University Press, 2008.

28. Beaton DE, Bombardier C, Guillemin F, Ferraz MB. Guidelines for the process of cross-cultural adaptation of self-report measures. Spine 2000;25:3186-91.

Correspondance et offprints : Marie-Paule GUSTIN, Département de Santé Publique; Faculté de Pharmacie (Institut des Sciences Pharmaceutiques et Biologiques) de Lyon, 8 avenue Rockefeller, 69373 Lyon cedex 08, France.

Mailto : gustin@univ-lyon1.fr 


\section{ANNEXE 1}

\section{R-SPQ-2F version française avec les items regroupés par dimension}

\begin{tabular}{|c|c|c|}
\hline SDD & $\mathrm{n}^{\circ}$ item & Libellé de l'item \\
\hline MP1 & 1 & J'éprouve un sentiment de profonde satisfaction personnelle quand j'étudie. \\
\hline MP2 & 5 & Potentiellement n'importe quel sujet peut être très intéressant, une fois que je m’y mets. \\
\hline MP3 & 9 & $\begin{array}{l}\text { Etudier la matière des cours universitaires peut être parfois aussi stimulant qu'un bon roman ou un } \\
\text { bon film. }\end{array}$ \\
\hline MP4 & 13 & Je travaille beaucoup pour mes études parce que je trouve le contenu intéressant. \\
\hline MP5 & 17 & J'arrive aux cours avec en tête des questions pour lesquelles j'aimerais des \\
\hline SP1 & 2 & $\begin{array}{l}\text { Pour être satisfait, je dois travailler suffisamment sur un sujet afin de pouvoir établir mes propres } \\
\text { conclusions. }\end{array}$ \\
\hline SP2 & 6 & $\begin{array}{l}\text { La plupart des nouveaux sujets m'intéressent, et je consacre du temps supplémentaire pour } \\
\text { compléter les informations sur ces sujets. }\end{array}$ \\
\hline SP3 & 10 & Je m'évalue moi-même sur les thèmes importants jusqu'à ce que je les comprenne complètement. \\
\hline SP4 & 14 & $\begin{array}{l}\text { Je passe beaucoup de mon temps libre à approfondir les thèmes intéressants qui ont été présentés } \\
\text { dans les différents cours. }\end{array}$ \\
\hline SP5 & 18 & Je m'assure d'avoir regardé la plupart des lectures conseillées qui accompagnent les cours. \\
\hline MS1 & 3 & Mon but est de réussir aux examens* en travaillant le moins possible. \\
\hline MS2 & 7 & Je ne trouve pas les cours très intéressants, donc je travaille le moins possible. \\
\hline MS3 & 11 & $\begin{array}{l}\text { Pour réussir les examens*, je m'en sors en mémorisant des parties clé plutôt qu'en essayant de les } \\
\text { comprendre. }\end{array}$ \\
\hline MS4 & 15 & $\begin{array}{l}\text { Je ne trouve pas utile d'étudier un sujet en profondeur : cela embrouille et fait perdre du temps, alors } \\
\text { qu'il suffit d'en avoir une connaissance momentanée. }\end{array}$ \\
\hline MS5 & 19 & Je ne vois pas l'intérêt d'apprendre la matière du cours qui ne sera probablement pas à l'examen*. \\
\hline SS1 & 4 & Je n'étudie sérieusement que ce qui m’est donné en cours ou ce qui est au programme. \\
\hline SS2 & 8 & $\begin{array}{l}\text { J'apprends certaines choses par répétition, en y revenant encore et encore jusqu'à ce que je les sache } \\
\text { par cœur même si je ne les comprends pas. }\end{array}$ \\
\hline SS3 & 12 & $\begin{array}{l}\text { Je limite mon travail à ce qui est demandé spécifiquement, car je pense qu'il n'est pas nécessaire de } \\
\text { faire des choses en plus. }\end{array}$ \\
\hline SS4 & 16 & $\begin{array}{l}\text { Les enseignants ne devraient pas s'attendre à ce que les étudiants passent beaucoup de temps à } \\
\text { étudier la matière dont chacun sait qu'elle ne sera pas testée. }\end{array}$ \\
\hline SS5 & 20 & $\begin{array}{l}\text { La meilleure façon de réussir aux examens est d'essayer de mémoriser les réponses aux questions } \\
\text { probables. }\end{array}$ \\
\hline
\end{tabular}

SSD : Sous-Dimension, MP : Motivation Profonde, SP : Stratégie Profonde, MS : Motivation de Surface,

SS: Stratégie de Surface

*Examen est remplacé par concours suivant le contexte 


\section{ANNEXE 2}

\section{R-SPQ-2F version française avec les items dans l'ordre de passation}

\begin{tabular}{|c|c|c|}
\hline SDD & $\mathrm{n}^{\circ}$ item & Libellé de l'item \\
\hline MP1 & 1 & J'éprouve un sentiment de profonde satisfaction personnelle quand j'étudie. \\
\hline SP1 & 2 & $\begin{array}{l}\text { Pour être satisfait, je dois travailler suffisamment sur un sujet afin de pouvoir établir mes propres } \\
\text { conclusions. }\end{array}$ \\
\hline MS1 & 3 & Mon but est de réussir aux examens* en travaillant le moins possible. \\
\hline SS1 & 4 & Je n'étudie sérieusement que ce qui m’est donné en cours ou ce qui est au programme. \\
\hline MP2 & 5 & Potentiellement nimporte quel sujet peut être très intéressant, une fois que je m'y mets. \\
\hline SP2 & 6 & $\begin{array}{l}\text { La plupart des nouveaux sujets m'intéressent, et je consacre du temps supplémentaire pour } \\
\text { compléter les informations sur ces sujets. }\end{array}$ \\
\hline MS2 & 7 & Je ne trouve pas les cours très intéressants, donc je travaille le moins possible. \\
\hline SS2 & 8 & $\begin{array}{l}\text { J'apprends certaines choses par répétition, en y revenant encore et encore jusqu'à ce que je les } \\
\text { sache par cœur même si je ne les comprends pas. }\end{array}$ \\
\hline MP3 & 9 & $\begin{array}{l}\text { Etudier la matière des cours universitaires peut être parfois aussi stimulant qu'un bon roman ou un } \\
\text { bon film. }\end{array}$ \\
\hline SP3 & 10 & Je m'évalue moi-même sur les thèmes importants jusqu'à ce que je les comprenne complètement. \\
\hline MS3 & 11 & $\begin{array}{l}\text { Pour réussir les examens*, je m'en sors en mémorisant des parties clé plutôt qu'en essayant de les } \\
\text { comprendre. }\end{array}$ \\
\hline SS3 & 12 & $\begin{array}{l}\text { Je limite mon travail à ce qui est demandé spécifiquement, car je pense qu'il n’est pas nécessaire de } \\
\text { faire des choses en plus. }\end{array}$ \\
\hline MP4 & 13 & Je travaille beaucoup pour mes études parce que je trouve le contenu intéressant. \\
\hline SP4 & 14 & $\begin{array}{l}\text { Je passe beaucoup de mon temps libre à approfondir les thèmes intéressants qui ont été présentés } \\
\text { dans les différents cours. }\end{array}$ \\
\hline MS4 & 15 & $\begin{array}{l}\text { Je ne trouve pas utile d'étudier un sujet en profondeur : cela embrouille et fait perdre du temps, } \\
\text { alors qu'il suffit d'en avoir une connaissance momentanée. }\end{array}$ \\
\hline SS4 & 16 & $\begin{array}{l}\text { Les enseignants ne devraient pas s'attendre à ce que les étudiants passent beaucoup de temps à } \\
\text { étudier la matière dont chacun sait qu'elle } \\
\text { ne sera pas testée. }\end{array}$ \\
\hline MP5 & 17 & J'arrive aux cours avec en tête des questions pour lesquelles j'aimerais des réponses. \\
\hline SP5 & 18 & Je m'assure d'avoir regardé la plupart des lectures conseillées qui accompagnent les cours. \\
\hline MS5 & 19 & Je ne vois pas l'intérêt d'apprendre la matière du cours qui ne sera probablement pas à l'examen*. \\
\hline SS5 & 20 & $\begin{array}{l}\text { La meilleure façon de réussir aux examens est d'essayer de mémoriser les réponses aux questions } \\
\text { probables. }\end{array}$ \\
\hline
\end{tabular}

SSD : Sous-Dimension, MP : Motivation Profonde, SP : Stratégie Profonde, MS : Motivation de Surface,

SS: Stratégie de Surface

*Examen est remplacé par concours suivant le contexte 


\section{ANNEXE 3}

\section{R-SPQ-2F version anglaise originale avec les items regroupés par sous-dimension}

\begin{tabular}{|c|c|c|}
\hline SBS & item & item label \\
\hline DM1 & 1 & I find that at times studying gives me a feeling of deep personal satisfaction. \\
\hline DM2 & 5 & I feel that virtually any topic can be highly interesting once I get into it. \\
\hline DM3 & 9 & I find that studying academic topics can at times be as exciting as a good novel or movie. \\
\hline DM4 & 13 & I work hard at my studies because I find the material interesting. \\
\hline DM5 & 17 & I come to most classes with questions in mind that I want answered. \\
\hline DS1 & 2 & $\begin{array}{l}\text { I find that I have to do enough work on a topic so that I can form my own conclusions before I am } \\
\text { satisfied. }\end{array}$ \\
\hline DS2 & 6 & $\begin{array}{l}\text { I find most new topics interesting and often spend extra time trying to obtain more information about } \\
\text { them. }\end{array}$ \\
\hline DS3 & 10 & I test myself on important topics until I understand them completely. \\
\hline DS4 & 14 & $\begin{array}{l}\text { I spend a lot of my free time finding out more about interesting topics which have been discussed in } \\
\text { different classes. }\end{array}$ \\
\hline DS5 & 18 & I make a point of looking at most of the suggested readings that go with the lectures. \\
\hline SM1 & 3 & My aim is to pass the course while doing as little work as possible. \\
\hline SM2 & 7 & I do not find my course very interesting so I keep my work to the minimum. \\
\hline SM3 & 11 & $\begin{array}{l}\text { I find I can get by in most assessments by memorising key sections rather than trying to understand } \\
\text { them. }\end{array}$ \\
\hline SM4 & 15 & I find it is not helpful to study topics in depth. \\
\hline SM5 & 19 & I see no point in learning material which is not likely to be in the examination. \\
\hline SS1 & 4 & I only study seriously what's given out in class or in the course outlines. \\
\hline SS2 & 8 & $\begin{array}{l}\text { I learn some things by rote, going over and over them until I know them by heart even if I do not } \\
\text { understand them. }\end{array}$ \\
\hline SS3 & 12 & $\begin{array}{l}\text { I generally restrict my study to what is specifically set as I think it is unnecessary to do anything } \\
\text { extra. }\end{array}$ \\
\hline SS4 & 16 & $\begin{array}{l}\text { I believe that lecturers shouldn't expect students to spend significant amounts of time studying } \\
\text { material everyone knows won't be examined. }\end{array}$ \\
\hline SS5 & 20 & I find the best way to pass examinations is to try to remember answers to likely questions. \\
\hline
\end{tabular}

SBS: SubScale, DM: Deep Motive, DS: Deep Strategy, SM: Surface Motive, SS: Surface Strategy 


\section{ANNEXE 4 - Les consignes}

\section{$R-S P Q-2 F$ version française}

Ce questionnaire porte sur votre rapport aux études et votre façon habituelle d'étudier en PACES.

Il n'existe pas une façon correcte d'étudier. Elle dépend de ce qui convient à votre style d'apprentissage et du cours que préparez. C'est pourquoi il est important que vous répondiez à chaque question aussi honnêtement que possible. Si vous pensez que votre réponse à une question dépend du sujet étudié, donnez la réponse que vous donneriez pour le(s) sujet(s) les plus important(s) pour vous.

Cocher pour chaque item l'option correspondant aux réponses suivantes :

A) Cet item n'est jamais ou seulement rarement vrai pour moi

B) Cet item est vrai de temps en temps pour moi

C) Cet item est vrai environ une fois sur deux pour moi

D) Cet item est souvent vrai pour moi

E) Cet item est toujours ou presque toujours vrai pour moi

Choisissez s'il vous plait la réponse la plus appropriée à chaque item. Cochez l'option qui correspond le mieux à votre première réaction. Ne passez pas trop de temps sur chaque item: votre première réaction est probablement la meilleure. S'il vous plait, répondez à chaque item.

$\mathrm{Ne}$ vous souciez pas de donner une bonne image de vous. Vos réponses sont CONFIDENTIELLES.

Nous vous remercions de votre coopération.

\section{R-SPQ-2F version originale en langue anglaise}

This questionnaire has a number of questions about your attitudes towards your studies and your usual way of studying.

There is no right way of studying. It depends on what suits your own style and the course you are studying. It is accordingly important that you answer each question as honestly as you can. If you think your answer to a question would depend on the subject being studied, give the answer that would apply to the subject(s) most important to you.

Please fill in the appropriate circle alongside the question number on the "General Purpose Survey/Answer Sheet". The letters alongside each number stand for the following response.

A) this item is never or only rarely true of me

B) this item is sometimes true of me

C) this item is true of me about half the time

D) this item is frequently true of me

E) this item is always or almost always true of me

Please choose the one most appropriate response to each question. Fill the oval on the Answer Sheet that best fits your immediate reaction. Do not spend a long time on each item: your first reaction is probably the best one. Please answer each item.

Do not worry about projecting a good image. Your answers are CONFIDENTIAL.

Thank you for your cooperation. 


\section{ANNEXE 5}

Diagramme des flux des étudiants inscrits en première année commune des études de santé (PACES) en septembre 2011 à l'Université Claude Bernard Lyon 1.

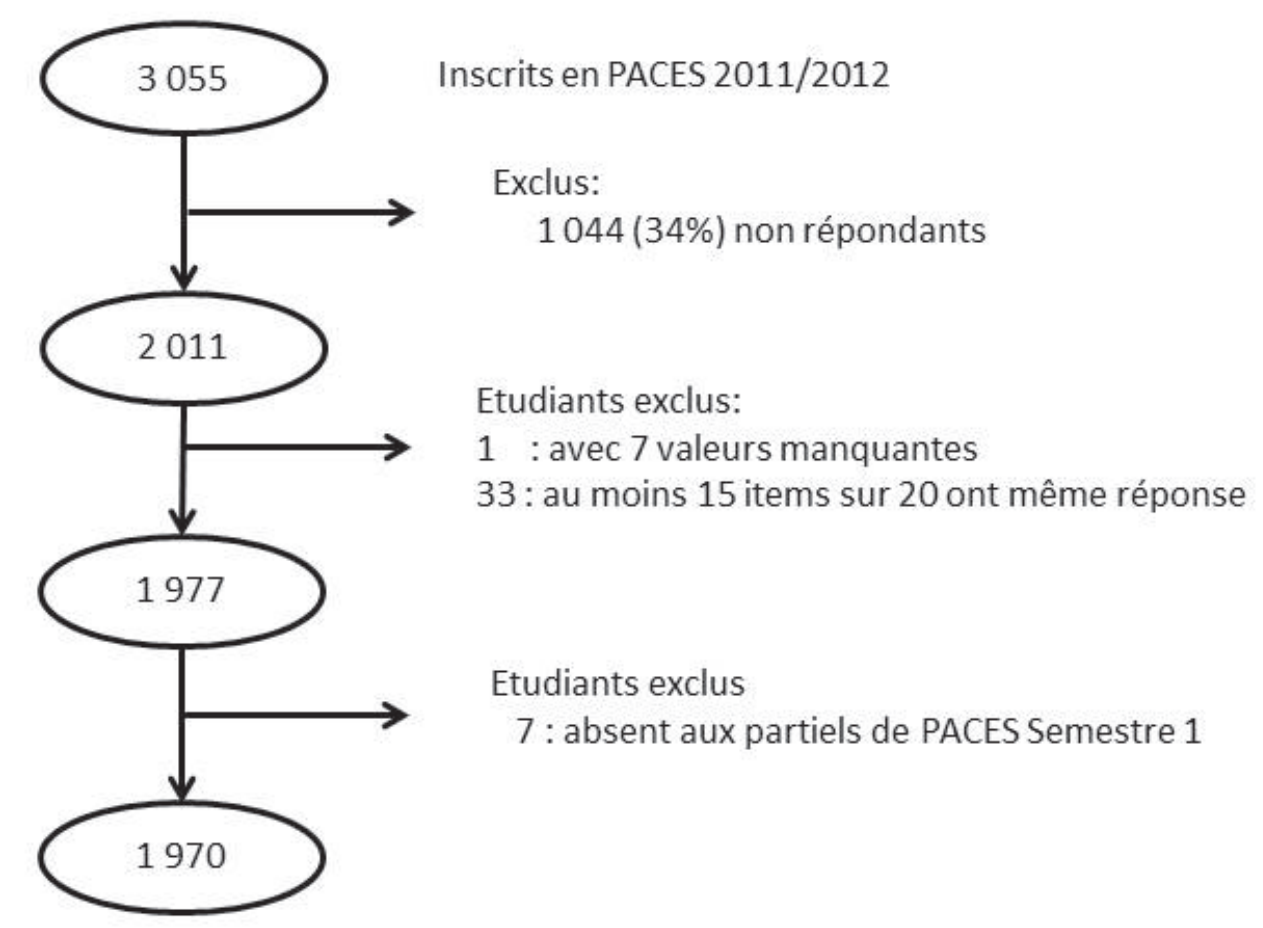

